\title{
ORIGINAL ARTICLE IS MENTAL HEALTH RESPONSIBLE FOR ANGER OUTBURSTS AND ULTIMATELY HYPERTENSION?
}

\author{
Mamoona Mushtaq ${ }^{1}$ \\ ${ }^{1}$ Govt MAO College, Lahore, Pakistan
}

Objectives: To explore the relationship of depression, anxiety, and stress (mental health) with anger dimensions and to study that if these mental states predict hypertension disease?

Methodology: Cross-sectional research design was used in the current research. Data was collected from 3 public sector hospitals of Lahore from May 15, 2019 to September 30, 2019. Sample of $(N=240)$ consecutive hypertensive patients with Mage $43.0707 \pm 7.99$ were recruited. Depression, Anxiety and Stress Scale (Lovibond \& Lovibond, 1995), and State Trait Anger Expression Inventory (Spielberger, 1988) were used for data collection. Descriptive statistics, logistic regression, and independent samples t-test were carried on for data analyses. Results: Significant positive correlation of depression, anxiety, and stress with anger dimensions was explore. Depression appeared as predictors of state anger, trait anger, anger in, anger control, and total anger $(\mathrm{p}<0.05)$. Anxiety emerged as predictor of state anger, trait anger, anger in, anger control, and total anger and stress as predictor of state anger, trait anger, anger in, anger control, and total anger. Significant gender differences appeared in depression, anxiety, stress, state anger, trait anger, anger in, anger control, and total anger $(\mathrm{p}<0.05)$.

Conclusion: Depression, anxiety, and stress have a significant relationship with anger and hypertension disease.

Keywords: Depression, anxiety, stress, anger, hypertension

Citation: Mushtaq M. Is mental health responsible for anger outbursts and ultimately hypertension?. Pak Heart J. 2021;54(04):339-343. DOI: https://doi.org/10.47144/phj.v54i4.2174

\section{INTRODUCTION}

The number of hypertensive adults is rapidly increasing, and it is estimated that the figure will touch 1.5 billion and the number will increase to $30 \%$ by 2025. Mostly, hypertensive patients live in middleand low-income countries. ${ }^{1}$ It is also reported that 1 every 4 men and 1 in every 5 women are suffering from hypertension and less than 1 in every 5 persons with hypertension has health issues that are under control. It is also established that hypertension is a major cause of early death globally. ${ }^{1}$

Hypertension despite being a highly modifiable risk factor for cardiovascular diseases is responsible for great mortality globally than any other risk factor. ${ }^{2}$ It is considered a key health issue due to its significant relevance to other diseases. Non-modifiable risk factors of hypertension are old age, family history of hypertension, socio-economic status, and sedentary lifestyle. ${ }^{3}$ The etiology of hypertension remains poorly understood, the contribution of heredity and lifestyle factors is different in unexplained outcomes. ${ }^{4}$

Anger is a strong emotion that can be triggered in many ways and may affect various mental and physical dimensions. Anger is a universal human emotion and a natural reaction to various events that inevitably happen in daily life. Literature has reported that cardiovascular diseases such as hypertension are closely associated with the human emotion of anger.,
6 The significant role of anger in developing hypertension and the rise of blood pressure levels during anger is remarkable. ${ }^{5}$ If an individual is not allowed to release anger-related impulses and suppress them, it can cause the onset of hypertension. ${ }^{6}$ It is established that people with hypertension are less assertive to manage their anger.

Anger has many dimensions that affect the body equally but are different in nature and expression as described by Spielberger (1988). ${ }^{7}$ State-anger is described as a psycho-physiological state comprising of personal feelings that are different in emotion from mild resentment to severe anger and is associated with the activation of the autonomic nervous system. ${ }^{7}$ Traitanger pertains to a difference from individual to individual in its occurrence with which state-anger is experienced. Trait anger is a dispositional characteristic of an individual that can express state anger. ${ }^{8}$ Therefore, trait-anger may precisely be called intense fury with a high rate of frequency of occurrence. An anger expression refers to a behavioral response of an individual based on the feelings of anger experienced. There are three anger expression styles: anger-in, anger-out, and anger control. ${ }^{9}$ Angerin is suppressed and internalized anger. This is the attribute of a person how often he/she passes through but held in or suppresses anger. It refers to the frequency with which angry feelings of an individual 
are suppressed. The individual in front of authority cannot express anger and must hold on in the angry feelings which have a destructive influence on the body. Anger-out is externalized anger expressed toward an object or another person. Anger-out is the frequency with which a person expresses angry feelings toward other people or objects in the environment in the form of verbal behavior. Anger control is the attempt to appropriately control one's anger expression. ${ }^{10}$ Anger control assesses the frequency of trying to control the expression of anger by an individual. It is the inclination of a person to control anger experience even experiencing an angry situation. Finally, anger total reflects either the intensity of feeling or the frequency with which it is expressed. $^{7}$

Anger suppression can lead to the physical manifestations of hypertension, thereby significantly negatively affecting health. ${ }^{8}$ It is reported that people with hypertension usually suppress their anger-related impulses. Anger suppression is another reason which is associated with a significant rise in high blood pressure even in normal people. ${ }^{11}$ Anger can also contribute to the adoption of an unhealthy lifestyle (smoking, consumption of high caloric aliments, alcohol, and caffeine consumption). Stress is one of the medically unexplained factors and a preventable cause of hypertension and a highly negative mental state. Stress is often considered as both cause and effect of hypertension and its role in developing hypertension is imperative. ${ }^{9}$ Personal perfection, anxious concern and blame proneness are significant predictors of anger outbursts. ${ }^{10}$

Depression is widespread in hypertensive patients after the withdrawal of anger outbursts. ${ }^{11}$ There is evidence of anger suppression and anger control in depressed patients. ${ }^{12}$ Depression has also been established as significantly related to violent behavior especially towards their spouse. Overall patients with depression exhibit more anger suppression and anger expression. ${ }^{13}$ Anger was found significantly related to the severity of depression and people exhibit more anger expression and anger-suppression. ${ }^{14}$ Similarly, they also exhibit a high level of repressed aggression and covert hostility, and they do not express aggression. ${ }^{15}$ Patients with depression experience a greater level of anger suppressed anger and apprehension of expressing anger as compared to healthy controls. ${ }^{11}$

Research indicates that anxiety and anger are dangerous to the physical health of an individual. ${ }^{11}$ Anxiety is considered a major cause of developing anger in people with hypertension. ${ }^{12,13}$ The significant relationship of anxiety with anger expression has already been explained in many pieces of research. ${ }^{14}$ People with anxiety may experience certain emotional problems like crying or getting frustrated easily, which further led to anger arousing. ${ }^{15}$ Similarly, anxiety and stress are prevalent among hypertensive patients are fully understood. ${ }^{16}$

There has been a dramatic change in lifestyle and social interactions on the advent of internet technology and the industrial revolution. In recent years a trend has been observed for shifting from villages to big cities where the pace of work is faster than villages and small towns which induces stress. ${ }^{5,17}$

To the best knowledge of the researchers, only a small data is available on exploring the anger outbursts and further hypertension. The biomedical community in Pakistan is still resistant to acknowledge the importance of psychological risk factors in the etiology of hypertension. Anger may be the most significant risk factor for hypertension. Though the subject is not new in the West and USA but has not been addressed in Pakistan yet. Thus, it was decided to research to explore the relationship of psychological factors of depression, anxiety, and stress with anger among hypertensive patients, so that some therapeutic or intervention program could be run to reduce the presently existing number of hypertensive patients. The following hypotheses were generated from the literature review.

H1: Depression, anxiety, and stress will have a significant relationship with anger dimensions (state anger, trait anger, anger in, anger out, anger control, and anger total) in hypertensive patients.

H2: Depression, anxiety, stress would predict anger and its dimensions (state anger, trait anger, anger in, anger out, anger control, and anger total) in hypertensive patients.

H3: There will be differences in mean scores of depressions, anxiety, stress, state anger, trait anger, anger in, anger out, anger control, and anger total between hypertensive men and women.

\section{METHODOLOGY}

In the current research, the cross-sectional research design was employed. Sample of 240 consecutive hypertensive patients, men (149) women (91) $\left(M_{\text {age }}=\right.$ $43.07 \pm 7.99$ ) were recruited from three public sector hospitals. Sample was obtained from outdoor departments of the hospitals when the hypertensive patients used to come for their regular check-ups. Purposive sampling technique used for data collection. The data was collected from May 15, 2019 to 
September 30, 2019. Criterion settled to include hypertensive patients were (a) those who have confirmed diagnosis of hypertension and have been taking medicines (b) who can read and write Urdu language (c) and those who gave consent to participate in the study. A demographic information form was prepared to collect information regarding age, education, occupation, number of dependents, family living system, monthly income, monthly expenditures, working hours, weight, and family history of hypertension of the research participants. Depression, Anxiety and Stress Scale (Lovibond \& Lovibond, 1995) was employed to assess depression, anxiety, and stress among hypertensive patients. The scale consists of overall 42 items and three subscales of depression, anxiety, and stress. Authors have reported $\alpha=.91$ for depression scale, .84 for anxiety and .90 for stress. ${ }^{17}$ Each item has 4 option response from 0 (did not apply to me at all) to 3 (applied to me very much) based on the Likert scale (38). The respondents can obtain scores from 0 to 42 . The highest scores indicate more depression, anxiety, and stress. ${ }^{17}$ State-Trait Anger Expression Inventory (Spielberger, 1988) comprised of 44 items was used. It has six subscales which are state anger, trait anger, anger in, anger out, anger control, and total anger. Each item has four optional response scores from 1 (never) to 4 (very often) based on a 4-point Likert scale. The respondents can obtain overall scores from 44 to76. State Trait Anger Expression Inventory has high internal consistency with $\alpha=0.95$ (40). ${ }^{7}$ Adapted Urdu version of the scale was used in the current research with Cronbach alpha $=0.94 .{ }^{18}$

After obtaining approval of the study from the Research Review Committee Punjab University, Lahore, permission from the selected scales' authors was obtained for using the scales in the study. Formal consent was obtained from the participant. Eligibility criteria, response format, and clear instructions were provided in the form. Confidentiality of their responses was assured and anonymity was maintained. After completing the form, they were cordially thanked for their cooperation.

\section{RESULTS}

Pearson correlation was carried out to explore the relationship of Anger dimensions with Depression, Anxiety, and Stress. Multiple Regression analysis was used to explore predictors of different dimensions of Anger. Independent samples t-test was run to explore differences in psychological risk factors and Anger dimensions between hypertensive men and women.

Results given in Table 1 indicate a significant correlation of state anger with depression, anxiety and with stress in hypertensive patients. Similarly, other dimensions of anger were found to be significantly and positively correlated with depression, anxiety and stress in hypertensive patients $(\mathrm{p}<0.05)$.

Linear Regression analysis was employed with depression, anxiety, and stress as predictor variables and anger dimensions as dependent variables. Findings further indicate that depression, anxiety, and stress have a significant positive effect and predicted state anger, trait anger, anger in, anger control and anger. Anger out remained non-significant in relation to depression, and anxiety, but significant in relation with anger out in hypertensive patients.

Results given in Table 3 depict that there are significant gender differences in depression, anxiety, stress, state anger, trait anger, anger in, anger out, anger control, and in total anger between hypertensive men and women at $\alpha 0.05$. Results indicate that hypertensive men scored high on anxiety, stress, state anger, anger out and anger total than hypertensive women. Conversely, hypertensive women attained high scores on depression, trait anger, anger in, and anger control.

Table 1: Correlation of Anger and its dimensions with Depression, Anxiety, Stress, and mean, SD and reliability

\begin{tabular}{|l|c|c|c|c|c|}
\hline Variable & Depression & Anxiety & Stress & Mean \pm SD & $\boldsymbol{\alpha}$ \\
\hline Sate Anger & $0.43^{*}$ & $0.67^{*}$ & $0.77^{*}$ & $15.80 \pm 6.46$ & 0.90 \\
\hline Trait Anger & $0.76^{*}$ & $0.68^{*}$ & $0.87^{*}$ & $21.07 \pm 8.45$ & 0.91 \\
\hline Anger-in & $0.87^{*}$ & $0.67^{*}$ & $0.85^{*}$ & $17.64 \pm 7.78$ & 0.92 \\
\hline Anger-out & -0.09 & 0.16 & -0.04 & $14.42 \pm 5.20$ & 0.81 \\
\hline Anger-control & $0.70^{*}$ & $0.55^{*}$ & $0.74^{*}$ & $20.25 \pm 8.13$ & 0.86 \\
\hline Anger & $0.80^{*}$ & $0.74^{*}$ & $0.86^{*}$ & $115.31 \pm 31.63$ & 0.94 \\
\hline Depression & - & - & - & $27.58 \pm 14.18$ & 0.89 \\
\hline Anxiety & - & - & - & $23.87 \pm 11.02$ & 0.93 \\
\hline Stress & - & - & - & $31.69 \pm 13.97$ & 0.95 \\
\hline
\end{tabular}

Note, ${ }^{*} p<.05, \alpha=$ reliability coefficient $;$ SA = State Anger, TA = Trait Anger, A/IN = Anger-in, A/OUT = Anger-out, A/CON = Anger-control, $A N G=$ Anger

Table 2: Depression, anxiety, and stress as a predictor of dimensions of anger in hypertensive patients $(n=240)$

\begin{tabular}{|l|l|l|l|l|l|l|}
\hline & State anger & Trait anger & Anger in & Anger out & Anger control & Anger \\
\hline Variables & $\boldsymbol{B}(\boldsymbol{S E})$ & $\boldsymbol{B}(\boldsymbol{S E})$ & $\boldsymbol{B}(\boldsymbol{S E})$ & $\boldsymbol{B}(\boldsymbol{S E})$ & $\boldsymbol{B}(\boldsymbol{S E})$ & $\boldsymbol{B}(\boldsymbol{S E})$ \\
\hline Depression & $0.38^{*}(0.16)$ & $0.27^{*}(.10)$ & $0.46^{*}(.23)$ & $0.09(0.02)$ & $0.70^{*}(0.32)$ & $0.80^{*}(0.35)$ \\
\hline
\end{tabular}




\begin{tabular}{|l|l|l|l|l|l|l|}
\hline Anxiety & $0.46^{*}(0.19)$ & $0.34 *(.12)$ & $.044 *(.21)$ & $0.10(0.02)$ & $0.56^{*}(0.23)$ & $0.63 *(0.27)$ \\
\hline Stress & $0.51^{*}(0.22)$ & $0.45 *(.18)$ & $0.57 *(.24)$ & $0.16(0.03)$ & $0.74 *(0.34)$ & $0.79 *(0.35)$ \\
\hline $\mathrm{R}^{2}$ & $59 \%$ & $70 \%$ & $64 \%$ & .03 & $61 \%$ & $86 \%$ \\
\hline $\mathrm{F}$ & 16.05 & 38.57 & 24.48 & 0.39 & 20.31 & 75.60 \\
\hline
\end{tabular}

Note: $p<0.05 ; R^{2}=0.79, \beta=$ standardized beta coefficient, $S E=$ standard error

Table 3: Difference of depression, anxiety, stress, SA, TA, AX/IN, ANG/OUT, ANG/CON, and anger between hypertensive men and women $(n=240)$

\begin{tabular}{|l|c|c|c|c|c|c|}
\hline \multirow{2}{*}{ Variables } & Hypertensive & Hypertensive & t $(\mathbf{d f})$ & Cohen's & \multicolumn{2}{|c|}{$\mathbf{9 5}$ \% C. I } \\
\cline { 5 - 7 } & Men & Women & $(\mathbf{2 3 4})$ & $\mathbf{d}$ & $\mathbf{L L}$ & $\mathbf{U L}$ \\
\hline Depression & $23.22 \pm 5.51$ & $32.79 \pm 16.29$ & $-6.26^{*}$ & 0.93 & -7.87 & -15.01 \\
\hline Anxiety & $26.37 \pm 12.66$ & $20.25 \pm 6.60$ & $-4.13^{*}$ & 0.83 & -3.19 & -9.03 \\
\hline Stress & $35.68 \pm 16.01$ & $25.87 \pm 7.06$ & $-5.36^{*}$ & 0.78 & -6.20 & -13.41 \\
\hline SA & $17.37 \pm 7.09$ & $13.51 \pm 4.54$ & $-4.48^{*}$ & 0.64 & -2.16 & -5.55 \\
\hline TA & $18.37 \pm 5.97$ & $22.91 \pm 3.36$ & $-3.98^{*}$ & 0.57 & -2.29 & -6.77 \\
\hline AX/IN & $15.24 \pm 3.36$ & $20.29 \pm 9.38$ & $-3.86^{*}$ & 0.56 & -1.98 & -6.12 \\
\hline AX/OUT & $19.66 \pm 5.31$ & $12.63 \pm 4.50$ & $-4.35^{*}$ & 0.61 & -1.65 & -4.41 \\
\hline AX/CON & $18.26 \pm 9.45$ & $23.77 \pm 5.32$ & $-2.26^{*}$ & 0.32 & -.29 & -4.77 \\
\hline Anger Total & $123 \pm 35.41$ & $103 \pm 20.23$ & $-4.68^{*}$ & 1.17 & -11.38 & -27.94 \\
\hline
\end{tabular}

Note; $p<0.05 ; C . I=$ Class interval, $L L=$ lower limit, $U L=$ upper limit, Cohen's $d=$ power of the test, $S A=$ stat anger, $T A=$ trait anger, $A X / I n=$ anger in, $A X /$ Out = anger out, $A X / C o n=$ anger control

\section{DISCUSSION}

The finding of the first hypothesis of the study indicates that depression, anxiety, and stress have a significant relationship with anger dimensions and are significant predictors of state anger among hypertensive patients. Hypertensive patients showed a great amount of depression and anger in the current study. Depression is a state of complete hopelessness that elevates anger related impulses when the environment is provoking. After the analysis of this study, it may be concluded that depressed feelings bring about an angry temperament. When there is a high level of difference between the real self and the ideal self, people suffer from greater depression and resultantly anger feelings are generated. ${ }^{1,19}$ Psychoanalysts say that hypertensive patients remain in anxiety whether to discharge their anger-related impulses or not. The state of anxiety brings them to release their defenses and hold them for a long period, consequently, they develop hypertension. ${ }^{11}$ When anxiety is excessive, the intensity behind it determines whether it will create a normal or angry reaction. ${ }^{13,20}$ Similarly, stress gives birth to irritation and angry temperament and is often considered as both cause and effect of anger and hypertension. ${ }^{7}$ In both ways, its role in developing anger and hypertension is crucial. Stress has been considered a causal factor in affecting the cognition $^{20}$ and developing anger among hypertensive patients. ${ }^{6,4}$, The researchers examined that angry thought slow down the improvement of hypertension. Individuals who are in the habit of thinking about past anger arousing actions are at higher risk of the continued increase in their blood pressure. $^{21}$
Linear regression analysis is computed with depression, anxiety, and stress as predictor variables and dimensions of anger (state anger, trait anger, anger-in, anger-out, anger-control) as outcome variables. The findings of the second hypothesis of the study indicate that depression, anxiety, and stress have a significant positive effect on state anger, trait anger, anger-in, anger-out, anger-control, and anger among hypertensive patients. Depressive, anxious, and stressful feelings are positively correlated with anger and its dimensions. ${ }^{6}$ It is important to explain that a positive correlation occurs between depression and anger dimensions. ${ }^{3}$ Therefore, it can be concluded that negative states of depression, anxiety, and stress are major factors in creating an angry temperament.

Moreover, the findings based on the 3rd hypothesis were also supported and are consistent with the earlier findings. ${ }^{22}$ The researchers have reported a greater level of depression among hypertensive men as compared to women. Further, anxiety was significantly higher in hypertensive men and these findings are consistent with the earlier findings., ${ }^{2,6}$ Earlier studies have reported less anxiety in hypertensive women than men.

Globally, it is confirmed that anger is a strong risk factor of hypertension in men than in women. ${ }^{3}$, It is internationally confirmed that women are higher on trait anger as compared to women. ${ }^{23}$ Anger in was found to be significantly associated with women than men. ${ }^{11}$ Women in Pakistani culture are not encouraged to express their anger in front of men or other older family members. Therefore, anger suppression becomes a personality disposition or trait of anger in women. Existing literature has reported the relationship of depression, anxiety, stress, and anger with hypertension ${ }^{2}$ as well as the role of anger in 
developing hypertension, ${ }^{24}$ still, no such research has assessed the role of anger in developing hypertension. The present research project is a unique one which explored both negative affect and anger dimensions in hypertensive patients.

Present research paved the way towards the results of previously conducted researches on the significance of psychological risk factors of hypertension. There is research evidence that some researches have been conducted in America and Europe ${ }^{5}$ on exploring the predictors of anger among hypertensive patients, but the research evidence is scant in Pakistan. The present research is a milestone in this area and will provide directions for future research in Pakistan.

Some limitations were observed during the process and most important of them was the small sample size which may not be deemed a true representative of the entire hypertensive population. It may be a threat to the external validity of this research. One more limitation of the research was that the data was collected during intensely heated months of summer from the patients waiting for their turn of checkup. Weather can be a great factor in reporting more anger in hypertensive patients.

\section{CONCLUSION}

Mental states of depression, anxiety, and stress were found to be significantly correlated to anger development and outbursts in hypertensive patients.

\section{AUTHORS' CONTRIBUTION:}

MM contributed to the concept and design of study, collected data, analysis and interpretation drafting of the manuscript, and approved the manuscript.

Conflict of interest: Authors declared no conflict of interest.

\section{REFERENCES}

1. Hypertension. (2019). World Health Organization. Retrieved on July 27, 2020, from https://www.who.int/newsroom/fact-sheets/detail/hypertension

2. Bakris G, Ali W, Parati G. ACC/AHA versus ESC/ESH on Hypertension Guidelines: JACC Guideline Comparison. J Am Coll Cardiol. 2019;73(23):3018-26.

3. Jang M.H, Lee J, Kim AS. Gender specific role of anger expression in association with depression and health behavior in community-dwelling elders. J Korean Acad Psychiatr Ment Health Nurs. 2014;23:38.

4. Mahadir NB, Yusoff MF, Abdullah S, Musa KI, Yaacob NM, Mohamad MS, et al. Factors associated with the severity of hypertension among Malaysian adults. PLoS One. 2019;14(1):e0207472.

\section{Address for Correspondence:}

Dr. Mamoona Mushtaq, Govt MAO College, Lahore, Pakistan.

Email: mamoonashahid74@gmail.com
5. Hu B, Liu X, Yin S, Fan H, Feng F, Yuan J. Effects of psychological stress on hypertension in middle-aged Chinese: A cross-sectional study. Cardiological Clin. 2017;35(2):22-230.

6. Akinlua JT, Meakin R, Umar AM, Freemantle N. Current, prevalence pattern of hypertension in Nigeria: A Systematic Review. PloS One. 2015;10(10):e0140021.

7. Spielberger CD. (1988). Manual for the State-Trait Anger Expression Inventory (STAXI). Odessa, FL: Psychological Assessment Resources Inc.

8. Fava M, Rosenbaum JF, Pava JA. Anger attacks in unipolar depression: Clinical correlates and response to fluoxetine treatment. Am J Psychiatry 1993;150:1158-63.

9. Dastan I, Erem A, Cetinkaya V. Urban and rural differences in hypertension risk factors in Turkey. Anatol $\mathrm{J}$ Cardiol. 2017;18(1):39-47.

10. Kim AS, Jang MH, Park KH, Min JY. Effects of self-efficacy, depression, and anger on health-promoting behaviors of Korean elderly women with hypertension. Int J Environ Res Public Health. 2020;17(17):6296.

11. Han A., Won J., Kim O., Lee S.E. Anger Expression Types and Interpersonal Problems in Nurses. Asian Nursing. Res. 2015;9:146-51.

12. Oh YH. The Health status of older Koreans and policy considerations. Health Welf. Policy Forum. 2015;223:29-39.

13. Schlomann P, Schmitke J. Lay beliefs about hypertension: An interpretive synthesis of the quality research. J Am Acad Nurse Pract. 2007;19(7):358-67.

14. Su M, Zhang QL, Bai XK, Wu CQ, Li YT, Mossialos E, et al. Availability, cost, and prescription patterns of anti-hypertensive medications in primary health care in China: A nation-wide crosssectional survey. Lancet. 2017;390(10112):2559-68.

15. Hu L, Huang X, You C, Li J, Hong K, Li P, et al. Prevalence and Risk Factors of Prehypertension and Hypertension in Southern China. PLoS One 2017;12:e0170238.

16. Gordon AM, Mendes WB. A large-scale study of stress, emotions, and blood pressure in daily life using a digital platform. Proc Natl Acad Sci U S A. 2021;118(31):e2105573118.

17. Lovibond SH, Lovibond PF. Manual for the Depression Anxiety Stress Scales. (2nd. Ed.) Sydney: Psychology Foundation, (1995).

18. Mushtaq M, Najam N. Factor structure of state trait anger expression inventory in urdu: a systematic review study of hypertensive patients. Pak J Psychol. 2014;45(1):17-43.

19. Crosswell AD, Whitehurst L, Mendes WB. Effects of acute stress on cognition in older versus younger adults. Psychol Aging, 2021;36:241-51.

20. Giena VP, Thongpat S, Nitirat P. Predictors of health-promoting behavior among older adults with hypertension in Indonesia. Int $\mathbf{J}$ Nurs Sci. 2018;5:201-5

21. Sadeghi B, Mashalchi H, Eghbali S, Jamshidi M, Golmohammadi M, Mahvar T. The relationship between hostility and anger with coronary heart disease in patients. J Educ Health Promot. 2020;9:223.

22. Cheng F, Lin P, Wang Y, Liu G, Li L, Yu H, et al. Type D personality and coronary atherosclerotic plaque vulnerability: The potential mediating effect of health behavior. J Psychosom Res. 2018;108:54-60.

23. Rodgers JL, Jones J, Bolleddu SI, Vanthenapalli S, Rodgers LE, Shah K, et al. Cardiovascular Risks Associated with Gender and Aging. J Cardiovasc Dev Dis. 2019;6:19.

24. Riaz M, Shah G, Asif M, Shah A, Adhikari K, et al. Factors associated with hypertension in Pakistan: A systematic review and meta-analysis. Plos One, 2021;16(1):e0246085. 